\title{
Catalase epitopes vaccine design for Helicobacter pylori: A bioinformatics approach
}

\author{
Niloufar Rashidi, Sharareh Moghim, Jamshid Fagheri and Hajieh Ghasemian Safaei* \\ Department of Microbiology, Faculty of Medicine, Isfahan University of Medical Sciences, Hezar jarib Street, Isfahan, \\ Iran. \\ Accepted 13 June, 2011
}

\begin{abstract}
Bioinformatics tools are helpful for epitopes prediction directly from the genomes of pathogens in order to design a vaccine. Epitopes are sub-sequences of proteins (8 to 10 mer peptides) which bind to MHC to interact with the $T$ cell receptors and stimulate immune responses. Finding a suitable vaccine against Helicobacter pylori is necessary, because of high prevalence of the infection (25 to $90 \%)$. Moreover, this bacteria has been classified as a grade I carcinogen by WHO since 1994. Catalase, an important enzyme in the virulence of $\boldsymbol{H}$. pylori, could be a suitable candidate for vaccine design because it is highly conserved, which is important for the survival of $\mathrm{H}$. pylori; it is expressed in high level and it is exposed on the surface of the bacteria. In this study, we designed epitope-based vaccine for catalase specific regions of $H$. pylori by means of immunobioinformatic tools. $H$. pylori (26695) catalase has been compared with human catalase in order to select specific regions. Afterwards, epitopes of catalase were determined by propred software. Among predicted epitopes, three epitopes were selected including, MVNKDVKQTT, VLLQSTWFL and FHPFDVTKI. Three candidates out of 51catalase antigen epitopes had the highest score for reactivating with MHC II MHC in propred software. The candidate epitopes for vaccine design should be rather a composition of considering epitopes: MVNKDVKQTTKKVLLQSTWFLKKFHPFDVTKI. In this manner, 39 of 51 alleles of MHC class II were involved and stimulated T-cell responses. We believe prediction of catalase epitopes by the immunoinformatics tools would be valuable for developing new immuoprophylatic strategy against $\boldsymbol{H}$. pylori infection.
\end{abstract}

Key words: Helicobacter pylori, catalase, epitopes.

\section{INTRODUCTION}

Importance of epitopes and bioinformatics tools

New genome analysis tools based on bioinformatics and immunoinformatics technologies help us to select the correct antigen or epitope of interest directly from the genomes of pathogens in order to design a vaccine (De Groot et al., 2001; Moise et al., 2008; Zhou et al., 2009).

To develop an epitope-based vaccine, it is necessary to detect immunostimulatory patterns. Protective immune responses may be generated based on a single immunedominant or more epitopes (De Groot and Berzofsky, 2004). Thus, it is important to choose epitopes derived

${ }^{\star}$ Corresponding author. E-mail: ghasemian@med.mui.ac.ir. Tel: 983117922469. Fax: 983116688597. from conserved peptide sequences (Khan et al., 2006). T-cell responses are stimulated by the presence of short peptides bound on the surface of antigen presenting cells and binding to major histocompatibility complex (MHC) molecules (De Groot and Berzofsky, 2004).

It is important to consider the protein antigen sequences that function at T-cell epitopes for successful vaccine formulations (Sette et al., 2001; Brusic and August, 2004; Khan et al., 2006). These tools allow us to decide on epitopes or sub-sequences of proteins that interface with the T-cells of the host and predict the MHC binder (De Groot and Berzofsky, 2004). Such tools let the scanning of genome-derived protein sequences for T-cell epitopes and the 8 to 10 mer peptides that bind to MHC and interact with the $T$ cell receptor, stimulate $T$-cell response. These epitopes prediction tools are proved to be very useful, since they significantly reduce the time and 
effort implicated in screening probable epitopes, mainly for pathogens that do not currently present available vaccines and still needs to be further investigated (De Groot and Berzofsky, 2004; Shi et al., 2007; Moise et al., 2008; Zhou et al., 2009). A usual epitope-based vaccine build contains epitopes inserted repeatedly in the construct, with or without intervening spacer amino acids (De Groot et al., 2001; Zhou et al., 2009; Liu et al., 2004). The advantages of this kind of vaccine are: stimulating specific immune responses against conserved sequences, increasing safety and increased strength and extent by the application of practically engineer epitopes (Shi et al., 2007).

Selection of candidate protein sequences can be followed by the use of computerized (in silico) software to recognize regions of the proteins that are probable immunogenic. These peptides, representing the epitopes, could be synthesized and their ability to stimulate immune response could be monitored, in vitro, by using prepared T-cells from patients (De Groot and Berzofsky, 2004).

\section{Helicobacter pylori infection and antigens}

Several microbial agents that cause considerably high rates of mortality and diseases are still waiting for vaccines. One of them is Helicobacter pylori $(H$. pylori). There are several problems caused by $H$. pylori infection including: (1) the eradication is not well satisfied; (2) high re-infection rates of patients; (3) high amount of drug dose for treatment; (4) antibiotics resistance (5) high prevalence (80 to 90\%) of infected populations in developing countries (Farshad et al., 2009; Ruggiero et al., 2003; Graham, 2000); (6) and classification as a grade 1 carcinogen by WHO (Radcliff et al., 1997; Schistosomes, 1994).

An antigen should have important features to be determined as a vaccine candidate: it should be expressed at the surface abundantly, in order to be easily recognized by immune system, and the sequences should be well conserved and represented as a key virulence factors (Ruggiero et al., 2003). Most of the vaccine of $\mathrm{H}$. pylori antigen investigated in mouse model was whole cell of bacteria, urease, heat shock protein, vacuolating cytotoxin and recently, catalase (Ruggiero et al., 2003; Liu et al., 2004).

Catalase could be a suitable candidate for vaccine design. It has very conserved domains, it is expressed in high levels ( $1 \%$ of the cell's total protein) (Harris et al., 2002), it is an important factor for survival of $H$. pylori in an environment rich in toxic oxygen species, found in both the cytosol and the periplasmic space and therefore, it is exposed on bacterial surface and could be easily identified by the immune system (Ramarao et al., 2000; Harris et al., 2002; Radcliff et al., 1997; Guy et al. 2005; Hazell et al., 1991).

\section{Immunity}

The mechanisms of defense against $H$. pylori infection are uncertain. Despite strong immune responses, the infection remains for a long time. It is suggested that the $H$. pylori can evade both innate and adaptive immune responses. The immune systems are triggered by $H$. pylori in nature but the responses are not good enough to eliminate this pathogen (Shi et al., 2007; Baldari et al., 2005). There are increasing evidences to hold up that $\mathrm{MHCll}$ restricted $\mathrm{CD} 4+$ cells play an essential role in defense mechanisms, more than antibodies. A successful immunotherapeutic or immunoprophylactic plan against H. pylori should include CD4+ Th cell epitopes (Shi et al., 2007; Ermak et al., 1998; Pappo et al., 1999).

Therefore, we believe that stimulating the immune response by vaccine, compared with that of the nature infection could be more effective against $H$. pylori (Shi et al., 2007). In this study, we decided to design epitopebased vaccine according to immunobioinformatic tools for the catalase of $H$. pylori.

\section{MATERIALS AND METHODS}

\section{Data collection and identification of specific sequences}

Complete sequence of $H$. pylori 26695 catalase were acquired from NCBI; accession code, NP-207669, 505 amino acid and used to identify the specific sequences of catalase in $H$. pylori (26695) and were compared with human catalase (NCBI, accession code: NP001743) and other strains of $H$. pylori using blast. The sequence of H. pylori 26695 catalase had 98 to $100 \%$ similarity with the catalase of other $\mathrm{H}$. pylori strains (25 strains) which are sequenced in NCBI such as $H$. pylori P12, G27, shi470, J99, ss1, 51,F32, 35A, 908, India 7, etc.

\section{Prediction of T-cell epitopes}

Several T-cell epitopes that are identified by more than one MHC and recognized by more than one $\mathrm{T}$ - cell clone are called 'promisecuous 'epitopes (De Groot et al., 2001; Khan et al., 2006). Different prediction methods according to biological information are considered for epitopes. In this study, we chose Propred software for prediction promiscuous T-cell epitopes (Singh and Raghava, 2001; Contini et al., 2008; Saltini et al., 2008). The propred web interface allows users to predict $\mathrm{MHC}$ class II binding regions in antigen sequence. $51 \mathrm{MHC}$ class II epitope-mapping matrices were developed at propred; therefore, it is helpful for the user in locating promiscuous or allele specific binding regions in a query antigen. The peptides (epitopes) predicted in this software, are nanomer. In each nanomer, there is an anchor or a starting residue. The 51 alleles of $\mathrm{MHC}$ class II in propred software were as following : DRB1:0101,0102,0301,0305,0306,0307,0308,0309,0311,0401,040 $2,0404,0405,0408,0410,0421,0423,0426,0701,0703,0801,0802,080$ $4,0806,0813,0817,1101,1102,1104,1106,1107,1114,1120,1121,112$ $8,1301,1302,1304,1305,1307,1311,1321,1322,1323,1327,1328,150$ 1,1502,1506, DRB5:0101,0105.

The propred software displayed predicted binders as blue colored region and $\mathrm{P} 1$ anchor or the starting residue of each predicted binding frame as red colored. This illustration is useful to highlight the locating promiscuous binding regions (Table 1). 


\section{RESULTS}

In this study, we selected three epitopes from unique specific and specific regions (Table 1). The first epitope was chosen from 1 to 8 , the second epitope from 32 to 44 and the third epitope from 267 to 288 regions of $H$. pylori catalase (Table2).

Of the 51 epitopes, the selected epitopes had the highest score for reactivating MHC class II in Propred software (Table 3). The first epitope (MVNKDVKQTT) contained 26, the second (VLLQSTWFL) contained 21 and the third (FHPFDVTKI) contained 16 alleles of the 51 MHCII (Table2).

According to blast with human catalase (NCBI, accession code: NP-001743), our result showed that the unique specific regions contained eight amino acids of the initial region from $\mathrm{N}$-terminal domain (MVNKDVKQ) and 19 amino acids of the final region from $\mathrm{C}$ - terminal domain (QKMMKDMHGKDMHHTKKKK). Other regions (shown as yellow boxes) were specific (Figure 1) as follows: sequences from 10 to 20 (TAFGAPVWDDN); 32 to 44(LLQSTWFLEKLAA); 209 to 243(ERFWVKFHFHTMQGVKHLTNEEAAEVRKYDPDS); 267 to 288 (PEEDAKKYRFHPFDVTKIWYLQ); 372 to 381(MQNGYYGSLO); 421-431(DDSDYYTQPG); 428-437(QPGDYYRSLP).

\section{DISCUSSION}

Prevalence of $H$. pylori infection is from $25 \%$ in developed countries to more than $90 \%$ in developing areas. H. pylori is a major cause of gastric carcinoma and lymphoma (Safaei et al., 2008; Rashidi and Ghasemian, 2005; Safaei et al., 2005; Mojtahedi et al., 2007; Safaei et al., 2010).

Therapeutic vaccines against $H$. pylori, which have been assayed so far, are whole bacteria lysate, recombinant subunit antigen vaccines and DNA vaccines but no major advance has been achieved (Zhou et al., 2009).

$\mathrm{CD}^{+}{ }^{+}$T-cells play important roles in producing protective immunity (Shi et al., 2007). Therefore, prediction antigenic epitopes in the context of MHC class II molecules expressed on APC (antigen presenting cell) recognized by $\mathrm{CD} 4^{+} \mathrm{T}$-cells can be use as a suitable tool for vaccination.

Catalase is a highly conserved enzyme, important for survival of the organism. There is 98 to $100 \%$ similarity between catalase of all Helicobacter species. This enzyme is expressed in high level and it is exposed on the surface of bacteria; therefore, it is a suitable antigen candidate for epitope vaccine evaluation (Radcliff et al., 1997). Therefore, in this study, we designed epitope vaccine for $H$. pylori catalase with Propred software. The suggested epitope should be rather a composition of selected three epitopes: MVNKDVKQTTKKVLLQSTWFLKKFHPFDVTKI. This epitope involved 39 out of 51 alleles of $\mathrm{MHC}$ class II and it stimulated T-cell responses. Lysines (KK) that is located between epitopes have some advantages: (1) cathepsine $\mathrm{B}$, a protease that has role in processing antigens for presentation by MHC II, considers $\mathrm{KK}$ as a target; (2) it is possible that a new epitope is formed from linkage of some epitopes; therefore, lysines $(\mathrm{KK})$ are an avoiding factor for this undesired event (Zhou et al., 2009). Guy et al. (2005) hypothesized four short fragments in N-terminal domain of $H$. pylori catalase (N1, N2, N3 and N4) and three short fragments in C-terminal domain which have shown a very low degree of sequence similarity with the human enzyme and can be introduced as specific regions for $H$. pylori protein. Our selected epitopes located in N1, N2 and N4 region was based on Guy's study. The first epitope was chosen from the amino acid residue of 1 to 20 which is the specific region of $\mathrm{N} 1$, the second epitope was from the amino acid residue of 30 to 44 which is the specific region of $\mathrm{N} 2$ and the third epitope was from the amino acid residue of 267 to 288 which is the specific region of N4 (Guy et al., 2005).

Sequences 10 to 20,372 to 381,421 to 431 and 428 to 437 are specific regions and 490 to 505 is the unique specific region according to blast (Figure 1). But there was no epitopes in them according to Propred software and could not be selected as a vaccine candidate.

In the unique specific and specific sequences of $H$. pylori catalase, 1 to 10,32 to 44,267 to 288,209 to 243 , MVNKDVKQTT, VLLQSTWFL, FHPFDVTKI, VKHLTNEEA and WVKFHFHTM epitopes were located (Table 3).

Our first epitope MVNKDVKQTT covered 26 alleles. The first epitope presented a high score (with ranking 1 to 3 among all of the scores in the epitopes) in the reaction with the following alleles: 0301, 0306, 0307, 0311, 0402, 0801, 0802, 0804, 0806, 0817, 1102, 1107, 1114, 1121, $1301,1322,1323,1327$ and 1328. Among the selected epitopes, the first one was the only one which could alone react with the alleles: 0301, 0305, 0309, 0801, 0802, 0804, 0806 and 0817, also, the MVNKDVKQTT reacted with alleles 0305, 0309, 0813, 1120 and 1302 and presented acceptable scores.

The second epitope (VLLQSTWFL) covered 21 alleles and in the reaction with six alleles $(0102,0402,0410$, 1301,1327 and 1328), it presented a high score. It is the only epitope that reacted with 0102 , for 0410 to have a high score among all the other epitopes. It also presented acceptable scores in other alleles.

The third one (FHPFDVTKI) contained 16 alleles. It scored high in the reaction with some alleles : 0101, $0408,0701,0703$ and 1502 . It is the only epitope that reacted with $0101,0408,0701$ and 0703 alleles and had a high score among all the other epitopes. Only this epitope among the five epitopes reacted with allele 1102 and presented acceptable score for it and other alleles.

The fourth epitope, WVKFHFHTM, covered 22 alleles. It presented a high score in the reaction with alleles as also for the first epitope $(1102,1121$ and 1322) and the third epitope (1502); presented high score. It had lower 
Table 1. Predicted epitopes of catalase enzyme by propred software.

\begin{tabular}{|c|c|c|c|}
\hline Allelle & $1-10$ & $31-39$ & 276-284 \\
\hline DRB1_0101 & MVNKDVKQTT & VLLQSTWFL & FHPFDVTKI \\
\hline DRB1_0102 & MVNKDVKQTT & VLLQSTWFL & FHPFDVTKI \\
\hline DRB1_0301 & MVNKDVKQTT & VLLQSTWFL & FHPFDVTKI \\
\hline DRB1_0305 & MVNKDVKQTT & VLLQSTWFL & FHPFDVTKI \\
\hline DRB1_0306 & MVNKDVKQTT & VLLQSTWFL & FHPFDVTKI \\
\hline DRB1_0307 & MVNKDVKQTT & VLLQSTWFL & FHPFDVTKI \\
\hline DRB1_0308 & MVNKDVKQTT & VLLQSTWFL & FHPFDVTKI \\
\hline DRB1_0309 & MVNKDVKQTT & VLLQSTWFL & FHPFDVTKI \\
\hline DRB1_0311 & MVNKDVKQTT & VLLQSTWFL & FHPFDVTKI \\
\hline DRB1_0401 & MVNKDVKQTT & VLLQSTWFL & FHPFDVTKI \\
\hline DRB1_0402 & MVNKDVKQTT & VLLQSTWFL & FHPFDVTKI \\
\hline DRB1_0404 & MVNKDVKQTT & VLLQSTWFL & FHPFDVTKI \\
\hline DRB1_0405 & MVNKDVKQTT & VLLQSTWFL & FHPFDVTKI \\
\hline DRB1_0408 & MVNKDVKQTT & VLLQSTWFL & FHPFDVTKI \\
\hline DRB1_0410 & MVNKDVKQTT & VLLQSTWFL & FHPFDVTKI \\
\hline DRB1_0421 & MVNKDVKQTT & VLLQSTWFL & FHPFDVTKI \\
\hline DRB1_0423 & MVNKDVKQTT & VLLQSTWFL & FHPFDVTKI \\
\hline DRB1_0426 & MVNKDVKQTT & VLLQSTWFL & FHPFDVTKI \\
\hline DRB1_0701 & MVNKDVKQTT & VLLQSTWFL & FHPFDVTKI \\
\hline DRB1_0703 & MVNKDVKQTT & VLLQSTWFL & FHPFDVTKI \\
\hline DRB1_0801 & MVNKDVKQTT & VLLQSTWFL & FHPFDVTKI \\
\hline DRB1_0802 & MVNKDVKQTT & VLLQSTWFL & FHPFDVTKI \\
\hline DRB1_0804 & MVNKDVKQTT & VLLQSTWFL & FHPFDVTKI \\
\hline DRB1_0806 & MVNKDVKQTT & VLLQSTWFL & FHPFDVTKI \\
\hline DRB1_0813 & MVNKDVKQTT & VLLQSTWFL & FHPFDVTKI \\
\hline DRB1_0817 & MVNKDVKQTT & VLLQSTWFL & FHPFDVTKI \\
\hline DRB1_1101 & MVNKDVKQTT & VLLQSTWFL & FHPFDVTKI \\
\hline DRB1_1102 & MVNKDVKQTT & VLLQSTWFL & FHPFDVTKI \\
\hline DRB1_1104 & MVNKDVKQTT & VLLQSTWFL & FHPFDVTKI \\
\hline DRB1_1106 & MVNKDVKQTT & VLLQSTWFL & FHPFDVTKI \\
\hline DRB1_1107 & MVNKDVKQTT & VLLQSTWFL & FHPFDVTKI \\
\hline DRB1_1114 & MVNKDVKQTT & VLLQSTWFL & FHPFDVTKI \\
\hline DRB1_1120 & MVNKDVKQTT & VLLQSTWFL & FHPFDVTKI \\
\hline DRB1_1121 & MVNKDVKQTT & VLLQSTWFL & FHPFDVTKI \\
\hline DRB1_1128 & MVNKDVKQTT & VLLQSTWFL & FHPFDVTKI \\
\hline DRB1_1301 & MVNKDVKQTT & VLLQSTWFL & FHPFDVTKI \\
\hline DRB1_1302 & MVNKDVKQTT & VLLQSTWFL & FHPFDVTKI \\
\hline DRB1_1304 & MVNKDVKQTT & VLLQSTWFL & FHPFDVTKI \\
\hline DRB1_1305 & MVNKDVKQTT & VLLQSTWFL & FHPFDVTKI \\
\hline DRB1_1307 & MVNKDVKQTT & VLLQSTWFL & FHPFDVTKI \\
\hline DRB1_1311 & MVNKDVKQTT & VLLQSTWFL & FHPFDVTKI \\
\hline DRB1_1321 & MVNKDVKQTT & VLLQSTWFL & FHPFDVTKI \\
\hline DRB1_1322 & MVNKDVKQTT & VLLQSTWFL & FHPFDVTKI \\
\hline DRB1_1323 & MVNKDVKQTT & VLLQSTWFL & FHPFDVTKI \\
\hline DRB1_1327 & MVNKDVKQTT & VLLQSTWFL & FHPFDVTKI \\
\hline DRB1_1328 & MVNKDVKQTT & VLLQSTWFL & FHPFDVTKI \\
\hline DRB1_1501 & MVNKDVKQTT & VLLQSTWFL & FHPFDVTKI \\
\hline DRB1_1502 & MVNKDVKQTT & VLLQSTWFL & FHPFDVTKI \\
\hline DRB1_1506 & MVNKDVKQTT & VLLQSTWFL & FHPFDVTKI \\
\hline DRB5 0101 & MVNKDVKQTT & VLLQSTWFL & FHPFDVTKI \\
\hline DRB5 0105 & MVNKDVKQTT & VLLQSTWFL & FHPFDVTKI \\
\hline
\end{tabular}

Blue region, predicted binders; red color, $\mathrm{P} 1$ anchor or the starting residue. 
Table 2. Number of reactivating MHC class II for selected epitopes according to Propred software.

\begin{tabular}{lcc}
\hline Epitope & Site of amino acid & Reactivating MHC \\
\hline MVNKDVKQTT & $1-10$ & 26 \\
VLLQSTWFL & $31-39$ & 21 \\
FHPFDVTKI & $276-284$ & 16 \\
\hline
\end{tabular}

Table 3. Comparison scores between the five epitopes according to propred software.

\begin{tabular}{|c|c|c|c|c|c|}
\hline Allele & $1^{*}$ & 2 & 3 & $4^{\star \star}$ & 5 \\
\hline DRB1-0101 & 0 & 5.33 & 14.50 & 4.33 & 0 \\
\hline DRB1-0102 & 0 & 22 & 14.50 & 0 & 8.33 \\
\hline DRB1-0301 & 40 & 22.74 & - & 15.79 & - \\
\hline DRB1-0305 & 23.8 & 2.20 & 9.89 & 13.19 & - \\
\hline DRB1-0306 & 36.36 & 4.55 & 0 & 25 & 10.23 \\
\hline DRB1-0307 & 36.36 & 4.55 & 0 & 25 & 10.23 \\
\hline DRB1-0308 & 36.36 & 4.55 & 0 & 25 & 10.23 \\
\hline DRB1-0309 & 29.47 & 12.21 & 18.95 & & \\
\hline DRB1-0311 & 36.36 & 4.55 & 0 & 25 & 10.23 \\
\hline DRB1-0401 & - & 15.12 & 8.14 & 11.63 & 30.23 \\
\hline DRB1-0402 & $\overline{25}$ & 40.62 & 11.46 & 23.96 & 8.33 \\
\hline DRB1-0404 & 0 & 25 & 23.86 & 4.55 & 32.95 \\
\hline DRB1-0405 & _- & 23.40 & 35.11 & 24.47 & 20.21 \\
\hline DRB1-0408 & - & 13.64 & 35.23 & 13.64 & 21.59 \\
\hline DRB1-0410 & 4.26 & 34.04 & 24.47 & 12.77 & 30.85 \\
\hline DRB1-0421 & - & 25.11 & 17.78 & 13.33 & 28.89 \\
\hline DRB1-0423 & $\overline{0}$ & 25 & 23.86 & 4.55 & 32.95 \\
\hline DRB1-0426 & _ & 15.12 & 8.14 & 11.63 & 30.23 \\
\hline DRB1-0701 & 11.21 & 36.38 & 49.14 & 37.93 & _ \\
\hline DRB1-0703 & 11.21 & 36.38 & 49.14 & 37.93 & - \\
\hline DRB1-0801 & 46.51 & - & 3.49 & 11.63 & - \\
\hline DRB1-0802 & 38.75 & - & 1.25 & 0 & - \\
\hline DRB1-0804 & 51.25 & $\overline{0}$ & 0 & _ & - \\
\hline DRB1-0806 & 58.14 & 6.98 & - & - & - \\
\hline DRB1-0813 & 29.89 & - & 33.33 & 8.05 & $\overline{0}$ \\
\hline DRB1-0817 & 45.54 & $3 . \overline{9} 6$ & 13.86 & 16.83 & _ \\
\hline DRB1-1101 & 10.84 & & 13.25 & 6.02 & $\overline{0}$ \\
\hline DRB1-1102 & 40.48 & 33.33 & 10.47 & 34.52 & 1.19 \\
\hline DRB1-1104 & 22.89 & 12.05 & 1.20 & 4.82 & 6.02 \\
\hline DRB1-1106 & 22.89 & 12.05 & 1.20 & 4.82 & 6.02 \\
\hline DRB1-1107 & 34.07 & - & - & 24.18 & 5.49 \\
\hline DRB1-1114 & 28.57 & 21.43 & 22.62 & 22.62 & _ \\
\hline DRB1-1120 & 35.23 & 31.36 & 31.82 & 32.95 & - \\
\hline DRB1-1121 & 40.48 & 33.33 & 10.71 & 34.52 & $1 . \overline{19}$ \\
\hline DRB1-1128 & 18.39 & 11.03 & 22.99 & 22.99 & - \\
\hline DRB1-1301 & 46.59 & 42.73 & 20.45 & 25 & - \\
\hline DRB1-1302 & 35.23 & 31.36 & 31.82 & 32.95 & - \\
\hline DRB1-1304 & 47.78 & 42.22 & 12.22 & 41.11 & - \\
\hline DRB1-1305 & 18.39 & 11.03 & 22.99 & 22.99 & - \\
\hline DRB1-1307 & 4.41 & 0 & - & 0 & $\overline{0}$ \\
\hline DRB1-1311 & 22.89 & 12.05 & 1.20 & 4.82 & 6.02 \\
\hline DRB1-1321 & 20.22 & 11.24 & 14.61 & 17.98 & - \\
\hline
\end{tabular}


Table 3. Continue.

\begin{tabular}{lccccc}
\hline DRB1-1322 & 40.48 & 33.33 & 10.71 & 34.52 & 1.19 \\
DRB1-1323 & 28.57 & 21.43 & 22.62 & 22.62 & - \\
DRB1-1327 & 46.59 & 42.73 & 20.45 & 25 & - \\
DRB1-1328 & 46.59 & 42.73 & 20.45 & 25 & - \\
DRB1-1501 & 11.22 & 23.06 & 31.63 & 39.59 & - \\
DRB1-1502 & - & 12.86 & 41.84 & 49.80 & 8.16 \\
DRB1-1506 & 11.22 & 23.06 & 31.63 & 39.59 & 18.37 \\
DRB5-0101 & - & 12.24 & 7.14 & 15.31 & - \\
DRB5-0105 & - & 12.24 & 7.14 & 15.31 & - \\
\hline
\end{tabular}

$1, \%$ highest score of MVNKDVKQTT; * 1, MVNKDVKQTT is a composition of two epitopes: MVNKDVKQT and VNKDVKQTT. Considered scores were related to epitope that had reaction with MHC or had higher score; $2, \%$ highest score of VLLQSTWFL; 3, \% highest score of FHPFDVTKI; 4, \% highest score of WVKFHFHTMQ; ${ }^{* *} 4-$ WVKFHFHTMQ, is a composition of two epitopes: WVKFHFHTM and VKFHFHTMQ. Considered scores were related to epitope that had reaction with MHC or has higher score; $5, \%$ highest score of VKHLTNEEA. Threshold is defined as the 'percentage of best scoring natural peptides'. Threshold setting in this study was $3 \%$. It predicts peptides in catalase sequence which belongs to the $3 \%$ best scoring natural peptides; yellow box, high score (1 to 3): the highest score with ranking of 1 to 3 among all of the scores in epitopes of specific and non specific regions; blue box, suitable score for the selected three epitopes; gray box, suitable score for the other two epitopes.

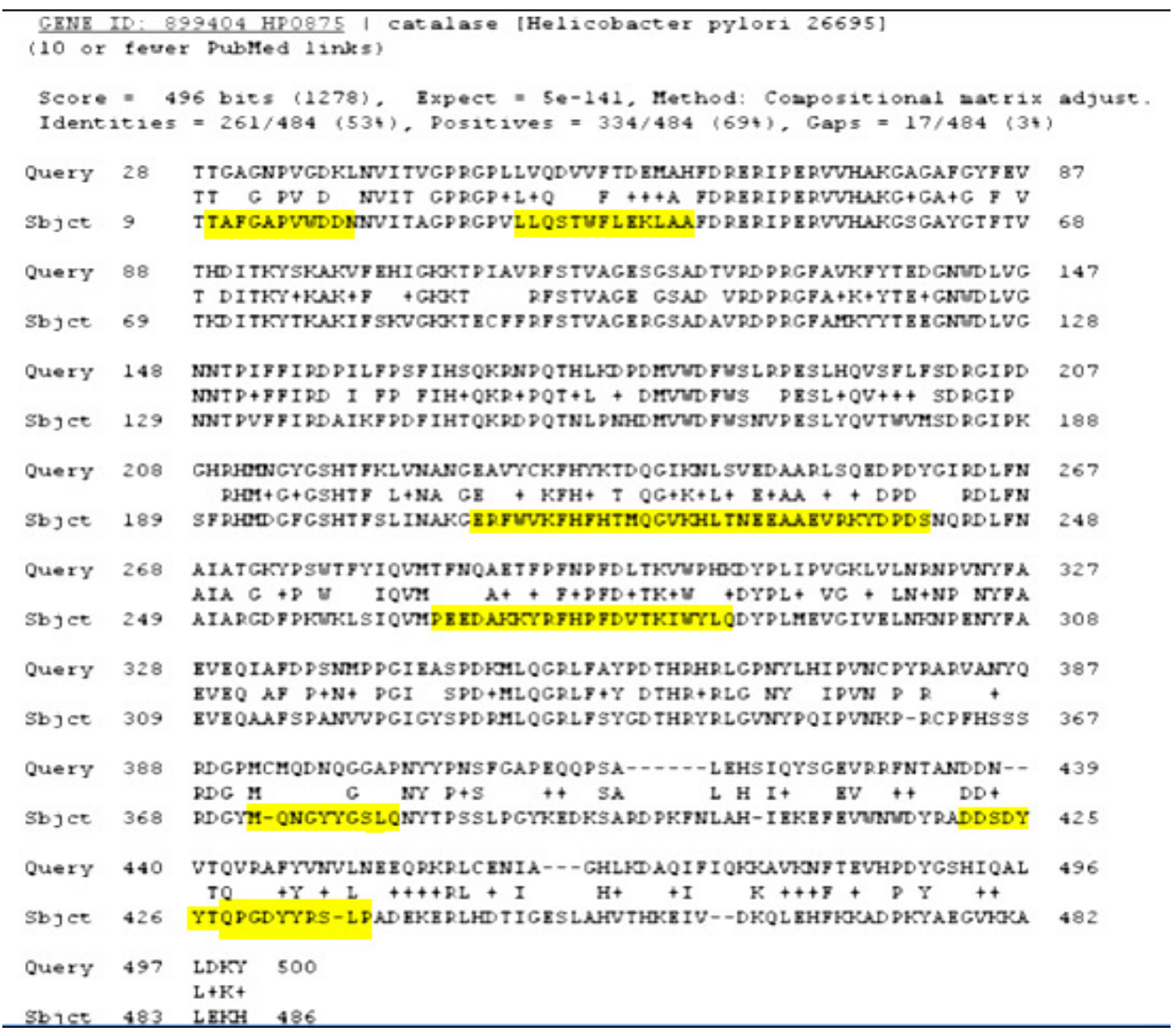

Figure 1. Result of blast search of specific sequences of H.pylori catalase (26695) and human catalase. Query, human catalase enzyme sequence; subject, $H$. pylori catalase enzyme sequence. 
score or the difference was not significant in reaction with the common alleles in other epitopes.

Although the reaction of the fifth epitope with three alleles was specific $(0401,0421$ and 0426) and its score in the reaction with two alleles (0404 and 0423) was high, this epitope, VKHLTNEEA covered only seven alleles which could not be enough for $\mathrm{T}$ cell responses in a variety of population.

Therefore, the three epitopes 1,2 and 3 was selected as: MVNKDVKQTTKKVLLQSTWFLKKFHPFDVTKI for laboratory investigation. Prediction of catalase epitopes in silico would be a valuable tool for developing new immuoprophylatic strategy against $H$. pylori infection. In addition, it could be helpful for the analysis of other $\mathrm{H}$. pylori antigens and other pathogens and provide a novel and usual progress for the design of epitope-based vaccines against many pathogens. It is crucial to study the cytokine production in vivo and in vitro for evaluation of the designed epitope vaccine. The antigenecity of designed epitopes for elucidating immune response as a vaccine candidate is under investigation in cell lines and animal models.

\section{ACKNOWLEDGEMENT}

This study was financially supported by the research Council of the Isfahan University of Medical Sciences.

\section{REFERENCES}

Baldari T, Lanzavecchia A, Telford JL (2005). Immune subversion by Helicobacter pylori. Trends Immunol. 26: 199-207.

Brusic V, August JT (2004). The changing field of vaccine development in the genomics era. 5: 597-600.

Contini S, Pallante M, Veibaesva S, Park MH, Chierakul N, Kim HS, Saltini C, Amicosante M (2008). A model of phenotypic susceptibility to Tuberculosis: Deficient insilico selection of Mycobacterium tuberculosis epitopes by HLA alleles. Sarcoidosisvasculitis and Diffuse. Lung. Disease, 25: 21-28.

De Groot AS, Bosma A, Chinai N, Frost J, Jesdale BM, Gonzalez MA, Martin W, Saint-Aubin C (2001). From genome to vaccine: in silico predictions, ex. vivo. verification. Vaccine, 19: 4385-4395.

De Groot AS, Berzofsky JA (2004). From genome to vaccine-new immunoinformatics tools for vaccine design. Methods, 34: 425428.

Ermak TH, Giannasca PJ, Nichols R, Myers GA, Nedrud J, Weltzi R, Lee C, Kleanthous H, Monath TP (1998). Immunization of mice with urease vaccine affords protection against Helicobacter pylori infection in the absence of antibodies and is mediated by .MHC. class IIrestricted responses. J. Exp. Med. 188: 2277.

Farshad S, Japoni A, Alborzi A (2009). Helicobacter pylori and Extradigestive Disorders in the Past 10 Years. IRCM. 1: 123-132.

Graham DY (2000). Therapy of Helicobacter pylori: current status and issues. Gastroenterology, 118: 2-8.

Guy B, Krell T, Sanchez V, Kennel A, Manin C, Sodoyer R (2005). Do Th1 or Th2 sequence motifs exist in proeins Identification of amphipatic immunomodulatory domains in Helicobacter pyloricatalase. Immunol. Lett. 96: 261-275.

Harris AG, HInds FE, Beckhouse AG, Kolesnikow T, Hazell SL (2002). Resistance to hydrogen peroxide in Helicobacter pylori: role of catalase (KatA) and Fur, and functional analysis of a novel gene product designated KatA-associated protein KapA (HP0874). Microbiology, 148: p. 3813.
Hazell SL, Evans JR. DJ, Graham DY (1991). Helicobacter pylori catalase. Microbiology, 137: p. 57.

Khan AM, Miotto O, Heiny AT, Salmon J, Srinivasan KN, Nascimento EJM, Marques ETA, Brusic V, Tan TW, August JT (2006). A systematic bioinformatics approach for selection of epitope-based vaccine targets. Cell. Immunol. 244: 141-147.

Liu XL, Li SQ, Liu CJ, Tao HX, Zhang ZS (2004). Antigen epitope of Helicobacter pylori vacuolating cytotoxin A, World J. Gastroenterol. 10: 2340-2343.

Moise L, Mcmurry JA, Pappo J, Lee DS, Moss SF, Martin WD, De Groot AS (2007). Identification of genome-derived vaccine candidates conserved between human and mouse-adapted strains of H. pylori. Hum. Vaccines, p. 4.

Mojtahedi A, Salehi R, Navabakbar F, Tamizifar H, Andalib A, Safaei H, Tavakkoli H, Farajzadega Z (2007) Apoptosis Induction on AGS. Gastric Adenocarcinoma and HEF Fibroblast Cell Lines by Wild Type and cagA or vacA Negative Helicobacter pylori Strains. J. Sci. Islam. Rep. Iran. 18: 203-208.

Pappo J, Torrey D, Castriotta L, Savinainen A, Kabok Z, Ibraghimov A (1999). Helicobacter pylori infection in immunized mice lacking major histocompatibility complex class I and class II functions. Infect. Immun. 67: 337.

Radcliff FJ, Hazell SL, Kolesnikow T, Doidge C, Lee A (1997). Catalase, a novel antigen for Helicobacter pylori vaccination. Infect. Immun. 65: 4668.

Ramarao N, Gray-Owen SD, Meyer TF (2000). Helicobacter pylori induces but survives the extracellular release of oxygen radicals from professional phagocytes using its catalase activity. Molecul. microbiol. 38: 103-113.

Rashidi N, Ghasemian SH (2005). in-house ellsa for diagnosis of hellcobacter pylori infection. J. Isfahan Med. Sch. 5-6: 91-95.

ruggiero $p$, peppoloni $s$, rappuoli $r$, del giudice $G$ (2003). The quest for a vaccine against Helicobacter pylori: how to move from mouse to man? Microb. Infect. 5: 749-756.

Safaei HG, Fazeli A, Tamizifar H,Rashidi N (2005). Comparison of Salivary Anti Helicobacte pylori IgG with Serum IgG and Bacteriological Tests in Detecting Helicobacter pylori Infections. J. Res. Med. Sci., 1: $1-5$.

Safaei HG, Tavakkoli H, Mojtahedi A, Salehei R, Soleimani B, Pishva E (2008). Correlation of cagA positive Helicobacter pylori Infection with clinical outcomes in Alzahra hospital, Isfahan. Iran. J. Res. Med. Sci., 13: 196-201.

Safaei HG, Havaei SA, Tavakkoli H, Eshaghei M, Navabakbar F, Salehei R, Emtiazi G, Heydari E, Saleh T, Irajian G (2010) .Relation of Bab A2 genotype of Helicobacter pylori infection with chronic active gastritis, duodenal ulcer and non-cardia active gastritis in Alzahra hospital Isfahan. Iran. Jundishapour J. Microbiol. 3: 93-98.

Saltini C, Pallante M, Puxeddu E, Contini S, Voorter CE, Dren M, Amicosant M (2008). M. avium binding to HLA-DR expressed alleles in silico: a model of phenotypic susceptibility to sarcoidosis. Sarcoidosis Vasculitis and diffuse lung diseases. 25: 100-116.

Schistosomes I (1994). Liver flukes and Helicobacter pylori. IARC. Monographs on the Evaluation of Carcinogenic Risks to Humans. Geneva WHO Publications, p. 61.

Sette A, Livingston B, Mckinney D, Appella E, Fikes J, Sidney J, Newman M, Chesnut R (2001). The development of multi-epitope vaccines: epitope identification, vaccine design and clinical evaluation. Biologicals, 29: 271-276.

Shi Y, Wu C, Zhou WY, Mao Biologicals. 29: 271-276. XH, GUO G, ZOU QM (2007). Identification of $\mathrm{H}-2 \mathrm{~d}$ restricted Th epitopes in Urease B subunit of Helicobacter pylori. Vaccine, 25: 2583-2590.

Singh H, Raghava GPS (2001). ProPred: prediction of HLA-Dr Binding site. Bioinformatics, 17: 1236-37.

Zhou WY, Shi Y, Wu C, Zhang WJ, MAO XH, Guo G, Li HX, Zou QM (2009). Therapeutic efficacy of a multi-epitope vaccine against Helicobacter pylori infection in BALB/c mice model. Vaccine, 27: 5013-5019. 